\title{
Tomographic Imaging and Monitoring of Buildings With Very High Resolution SAR Data
}

\author{
D. Reale, G. Fornaro, Senior Member, IEEE, A. Pauciullo, X. Zhu, Student Member, IEEE, and \\ R. Bamler, Fellow, IEEE
}

\begin{abstract}
Layover is frequent in imaging and monitoring with synthetic aperture radar (SAR) areas characterized by a high density of scatterers with steep topography, e.g., in urban environment. Using medium-resolution SAR data tomographic techniques has been proven to be capable of separating multiple scatterers interfering (in layover) in the same pixel. With the advent of the new generation of high-resolution sensors, the layover effect on buildings becomes more evident. In this letter, we exploit the potential of the 4-D imaging applied to a set of TerraSAR-X spotlight acquisitions. Results show that the combination of high-resolution data and advanced coherent processing techniques can lead to impressive reconstruction and monitoring capabilities of the whole 3-D structure of buildings.
\end{abstract}

Index Terms-Differential interferometric synthetic aperture radar (DInSAR), multidimensional SAR processing, SAR tomography, synthetic aperture radar (SAR), TerraSAR-X (TSX), 4-D SAR imaging.

\section{INTRODUCTION}

I NTERFEROMETRIC synthetic aperture radar (SAR) (InSAR) and differential InSAR (DInSAR), particularly multitemporal DInSAR, have been proven to be effective for accurate scatterer localization and monitoring of displacements [1], [2]. The high accuracy and spatial density of the measurements make these techniques cost effective compared to classical geodetic techniques, typically used in environmental risk monitoring.

The increase of the spatial resolution provides a tangible improvement in the monitoring capabilities: Most of the international space agencies have hence hugely invested in the launch of large bandwidth spaceborne SAR systems. The hardware improvement must be complemented by the development of processing techniques that are able to extract the highest possible information content from the data. In this sense, SAR

Manuscript received October 14, 2010; accepted November 25, 2010. Date of publication January 23, 2011; date of current version June 24, 2011. This work was supported in part by the European Union Integrated System for Transport Infrastructures Surveillance and Monitoring by Electromagnetic Sensing (ISTIMES) project and in part by the International Graduate School of Science and Engineering, Technische Universitaet Muenchen, Munich, Germany.

D. Reale, G. Fornaro, and A. Pauciullo are with the Institute for the Electromagnetic Sensing of the Environment (IREA), National Research Council (CNR), 80124 Napoli, Italy (e-mail: reale.d@irea.cnr.it; fornaro.g@ @irea.cnr.it; pauciullo.a@irea.cnr.it).

$\mathrm{X}$. Zhu is with the Technische Universität München, Lehrstuhl für Methodik der Fernerkundung, 80333 Munich, Germany (e-mail: xiaoxiang.zhu@bv. tum.de).

R. Bamler is with the Remote Sensing Technology Institute (IMF), German Aerospace Center (DLR), 82234 Oberpfaffenhofen, Germany, and also with the Technische Universität München, Lehrstuhl für Methodik der Fernerkundung, 80333 Munich, Germany (e-mail: richard.bamler@ dlr.de).

Digital Object Identifier 10.1109/LGRS.2010.2098845 tomography, also known as multidimensional (3-D and 4-D) imaging SAR (MDI-SAR), is recognized as a powerful technique that extends interferometry.

DInSAR and persistent scatterer interferometry (PSI) assume the presence of only a single (dominant) scattering center in each pixel. However, SAR images of complex scenarios are affected by the interference between the responses of scatterers located at different elevations (slant heights). Standard multipass interferometric techniques "look" for the matching between the received signal and the "multipass signature" of a scatterer: The interference of responses may hence lead to misdetection of persistent scatterers and to height, velocity, and time-series measurement inaccuracies.

The layover effect causes interference between the responses of different scatterers. Layover is particularly critical in urban areas which are characterized by a high density of scatterers distributed on vertical structures.

As briefly explained next, MDI-SAR allows the overcoming of the single scatterer assumption and has opened a new scenario in the 3-D target reconstruction and monitoring with SAR systems [3], [4]. On medium-resolution systems, MDI-SAR imaging has already been proven to be effective in separating and monitoring scatterers in layover [5], [6].

The new generation of high-resolution SAR sensors, such as TerraSAR-X (TSX) and the COSMO-SkyMed constellation, allows the systematic acquisition of data with spatial resolution reaching metric/submetric values. The preliminary analysis of these images in dense urban areas has indicated that the resolution improvement brings layover of vertical structures to be more pronounced. On high-resolution SAR data, the interference between scatterers on the ground and on buildings is more frequent, and it is distributed on more pixels than on data acquired by medium-resolution satellites (e.g., European Remote Sensing (ERS) satellite or ENVISAT): The tomographic approach is a tool that allows mitigating this problem [7]. Moreover, the higher the resolution, the higher are the expectations for 3-D reconstruction on vertical structures.

In this letter, we investigate the application of SAR tomography to a real data set of TSX spotlight images over the city of Las Vegas, NV. The characteristics of this data set allow clear demonstration of the potential and the advantages offered by the SAR tomography technique.

\section{LAYOVER AND TOMOGRAPHY}

The imaging mechanism of radar is measuring the distances (range) of the scatterers from the sensor. If two scatterers are 


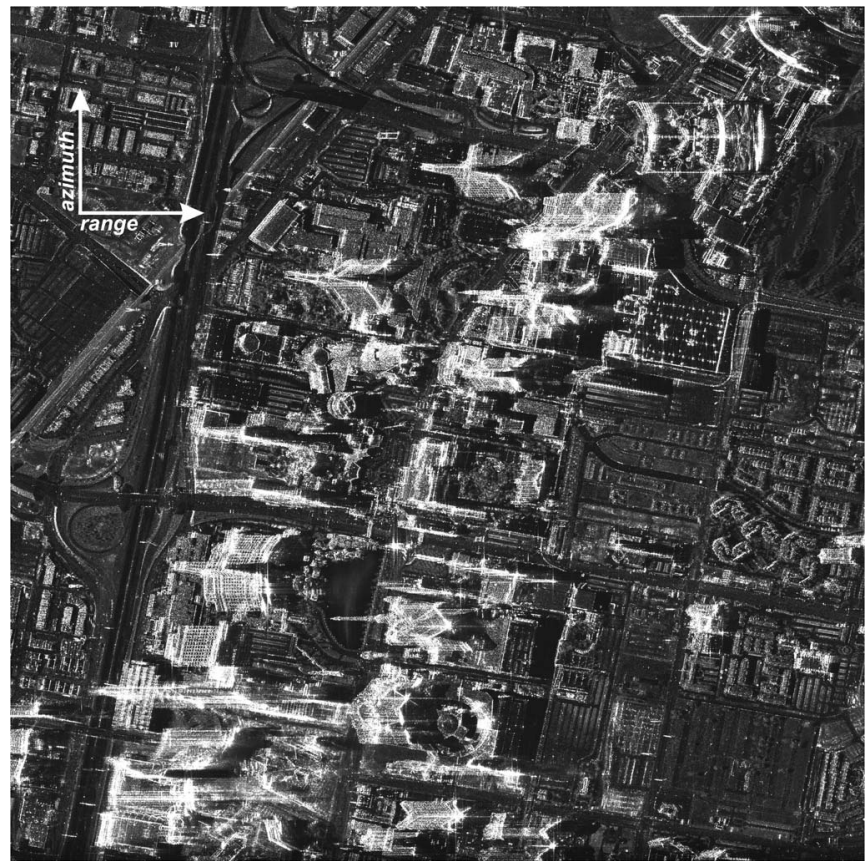

Fig. 1. Temporal multilook image of the spotlight TSX data set over the city of Las Vegas. The layover induced by the buildings is well recognizable.

located at different positions but share the same range, they are imaged into the same pixel: This effect is known as layover.

In the presence of a vertical structure, such as a building, the radar signal is affected by layover between the ground, the façade, and possibly the roof. To have an idea of the effects of layover, a data set of 25 TSX spotlight images from ascending orbits over the city of Las Vegas, NV, has been considered. This celebrated city, located over a flat area, includes a high number of tall structures. The multitemporal averaged amplitude image is shown in Fig. 1: Tall buildings are well visible, although "folded" onto the ground toward the sensor; hence, their responses interfere with those of the targets located below the structures.

The tomography principle is simple: By using SAR data acquired from different elevations, an antenna along the slant height direction can be synthesized. The synthesized array brings resolving capabilities on the backscattering distribution along the elevation direction, orthogonal to the radar line of sight, and hence, it leads to the possibility to separate contributions coming from scatterers with different elevations and interfering in the same pixel [5]. The tomographic technique (3-D imaging) has been extended also to the time direction: The differential-tomography technique (also known as 4-D, i.e., space-velocity imaging) allows the separation of interfering scatterers and the measurement of their (possibly) different velocities [3] and time series [4].

MDI-SAR exploits both amplitude and phase information to reconstruct, for each pixel in the spatial (i.e., azimuth/ range) domain, the backscattering distribution along the slant height/mean deformation velocity plane. This fact already allows the improvement of performance in terms of dominant persistent scatterer detection with respect to classical PSI that uses only phase information [8]. In this letter, we limited the tomographic analysis to single (dominant) and double scatterers.

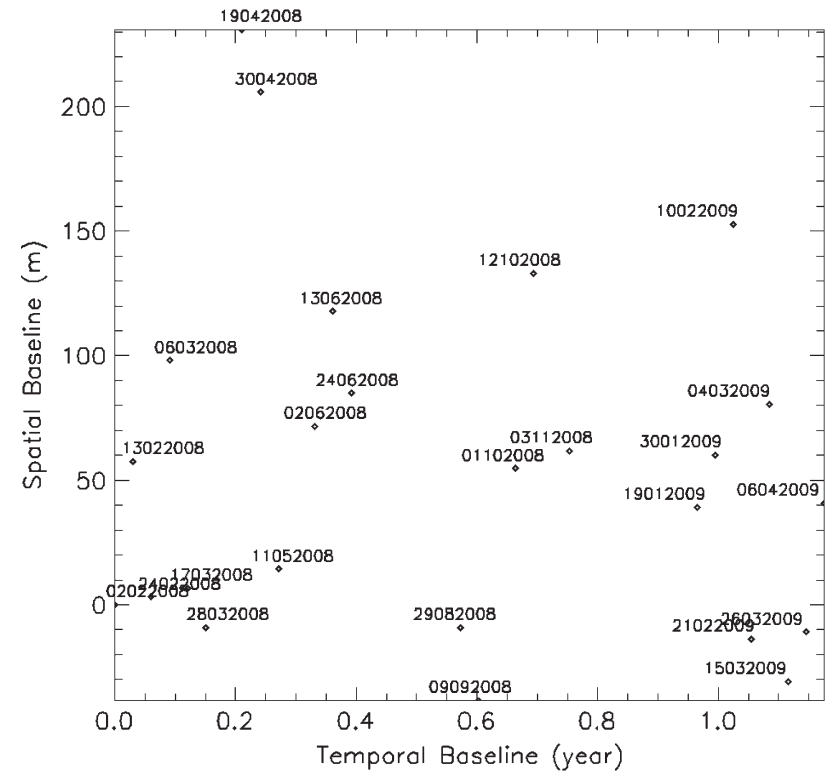

Fig. 2. Distribution of the acquisitions in the spatial/temporal baseline domain.

To search for single and double scatterers, we used the detection approach discussed in [9] and [10] based on the generalized likelihood ratio test. It exploits the detector for single scatterers in [9] in a sequential way and tests the energy contribution of the (possible) second scatterer after the cancellation of the dominant contribution: If this test declares the absence of the second scatterer, a second test on the presence of only one scatterer is carried out; see [10] for more details.

\section{EXPERIMENTAL RESULTS}

The TSX spotlight acquisition mode provides resolutions of $1.1 \mathrm{~m}$ in azimuth and $0.6 \mathrm{~m}$ in slant range. We applied the MDI technique to the area of Boulevard South, also known as "The Strip," where many of the largest hotels, casinos, and resorts are located. Almost all the images are acquired with the minimum repeat cycle of 11 days, from February 2008 to April 2009: Fig. 2 shows the baseline distribution. We note that, except for two acquisitions, the orbital tube is rather strict: The baseline span $(B)$ is only approximately $207 \mathrm{~m}$. This fact results in a poor slant height resolution of about $\delta_{s}=\lambda r / 2 B \cong 47 \mathrm{~m}$, corresponding to a height resolution of $\delta_{z}=\delta_{s} \sin (\vartheta) \cong 27 \mathrm{~m}$, where $\lambda, r$, and $\vartheta$ are the wavelength, the distance from the scene center, and the look angle, respectively. Superresolution SAR tomography techniques could limit the effects of this poor resolution [7], [12]: In this letter, however, we limited our analysis to the classical linear tomographic approach [6].

The data set was calibrated for atmospheric phase components estimated via the low-resolution multipass DInSAR approach in [13] before the tomographic processing.

We focused our analysis on the block of the Mirage Hotel and Casino. It presents a tall (about $100 \mathrm{~m}$ ) building surrounded by a lower flat structure (entertainment attractions) about 15-20 m over the street level.

Many features can be pointed out by comparing, in Fig. 3, the amplitude image of the area with an orthophotograph: 


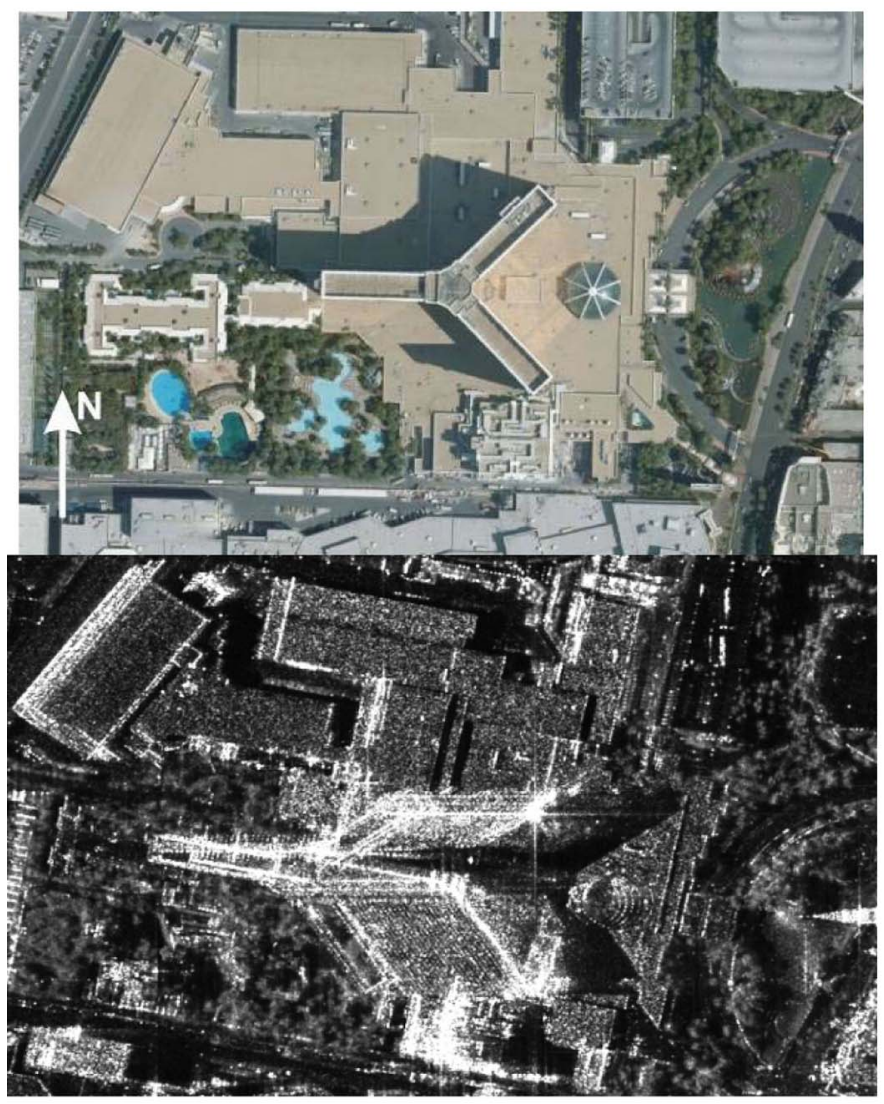

Fig. 3. (Top) Mirage Hotel image taken from Bing maps. (Bottom) TSX amplitude image.

1) the folding of the building toward the sensor due to the layover (the base of the Mirage hotel is almost vertically aligned in the Bing and TSX images); 2) the high range resolution distributes the response of the building over a large number of pixels; 3) the extremely high resolving capabilities of the TSX spotlight imaging that allows distinguishing floors on the southern façade.

\section{A. Single-Scatterer Analysis}

In Fig. 4 (top), we show the residual topography (i.e., the topography estimated after the subtraction of the external digital elevation model-in our case, Shuttle Radar Topography Mission) resulting from the MDI, followed by the single scatterer detection algorithm in [9], which tests the presence of a persistent scatterer based on energy content along the direction of the peak of the tomographic reconstruction. The building rising toward the sensor is well recognizable in the detected scatterers. As for previous analyses of TSX data [8], the density of the detected points is also impressive.

Some considerations are now in order: First, on the southern façade, many blue points corresponding to the ground are detected and are mixed to scatterers colored from green to red, corresponding to the vertical structure of the hotel. This fact testifies that the interference in the façade and ground is very likely. Second, in the upper right part of the image, two straight black strips (almost aligned to the azimuth) appear clearly. These areas correspond to two shadowing areas caused by small
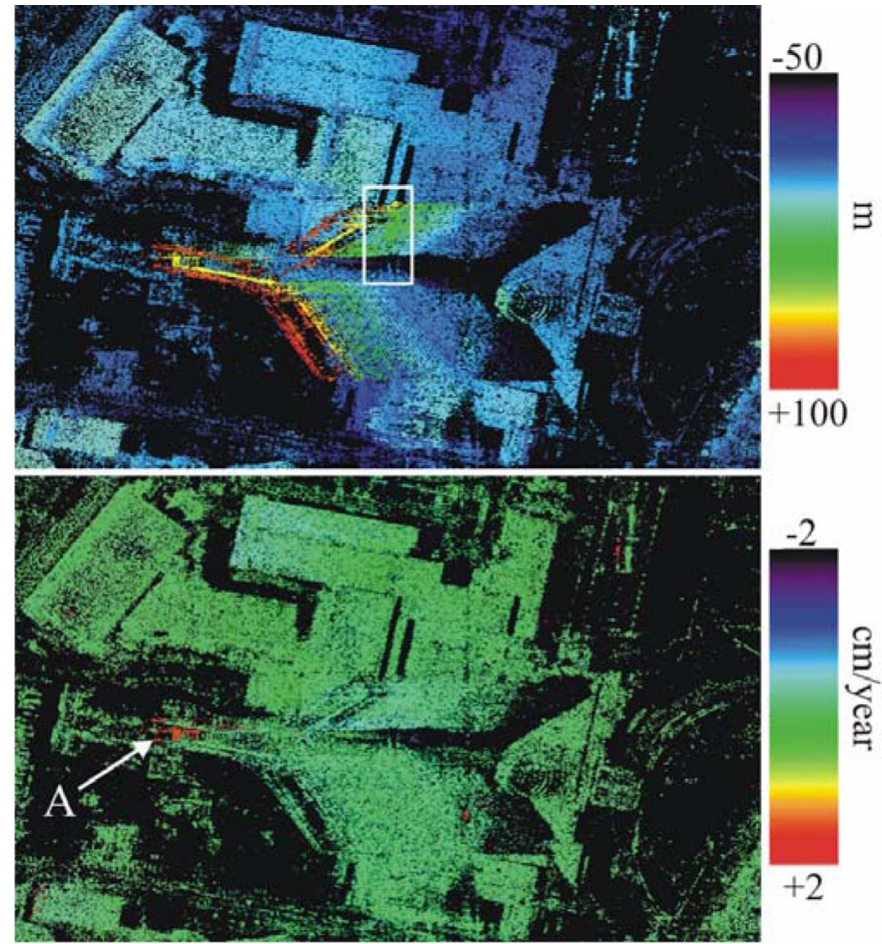

Fig. 4. (Top) Residual topography and (bottom) mean deformation velocity estimated by means of SAR tomography for the single-scatterer analysis.
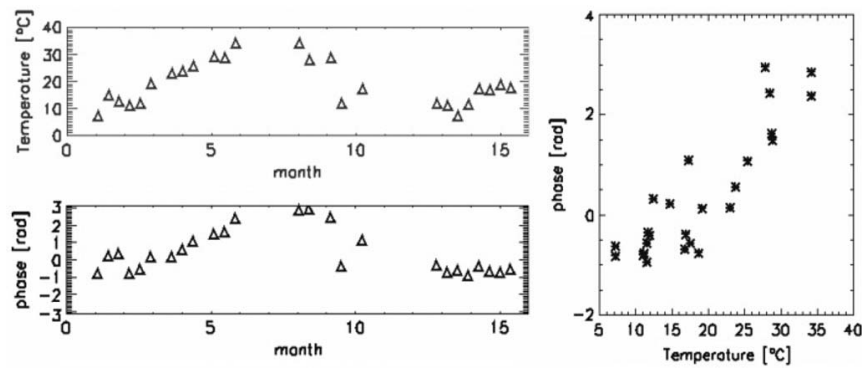

Fig. 5. (Upper left image) Daily averaged temperature of the area. (Lower left image) Residual phases after topography calibration for pixel A. (Right image) Scatter plot.

steps (a few meters high) on the roof of the surrounding structure. One of these shadow strips falls in the radar image areas under the layover of the north façade of the hotel. It is interesting to notice in this area the presence of a high density of scatterers (green pixels) on the part of the façade that falls over the shadowed strips (see the white box in Fig. 4): This high density is the result of the absence of any interference with the ground.

The deformation map presented at the bottom of Fig. 4 also shows an interesting phenomenon: While all the rest is stable, the roof appears moving toward the sensor at about $2 \mathrm{~cm} / y e a r$.

For one of these apparently inflating scatterer (A in Fig. 4), the phase signal obtained after the compensation of the topographic signature is shown in the lower left image in Fig. 5: This plot highlights the presence of a seasonal motion, and hence, the mean velocity is only in part able to explain this movement. The average daily temperatures of the area, provided by the University of Dayton database [14], are shown in the upper left image in Fig. 5. The high degree of correlation with the deformation is evident; see also the scatter plot in the image on the right. As 


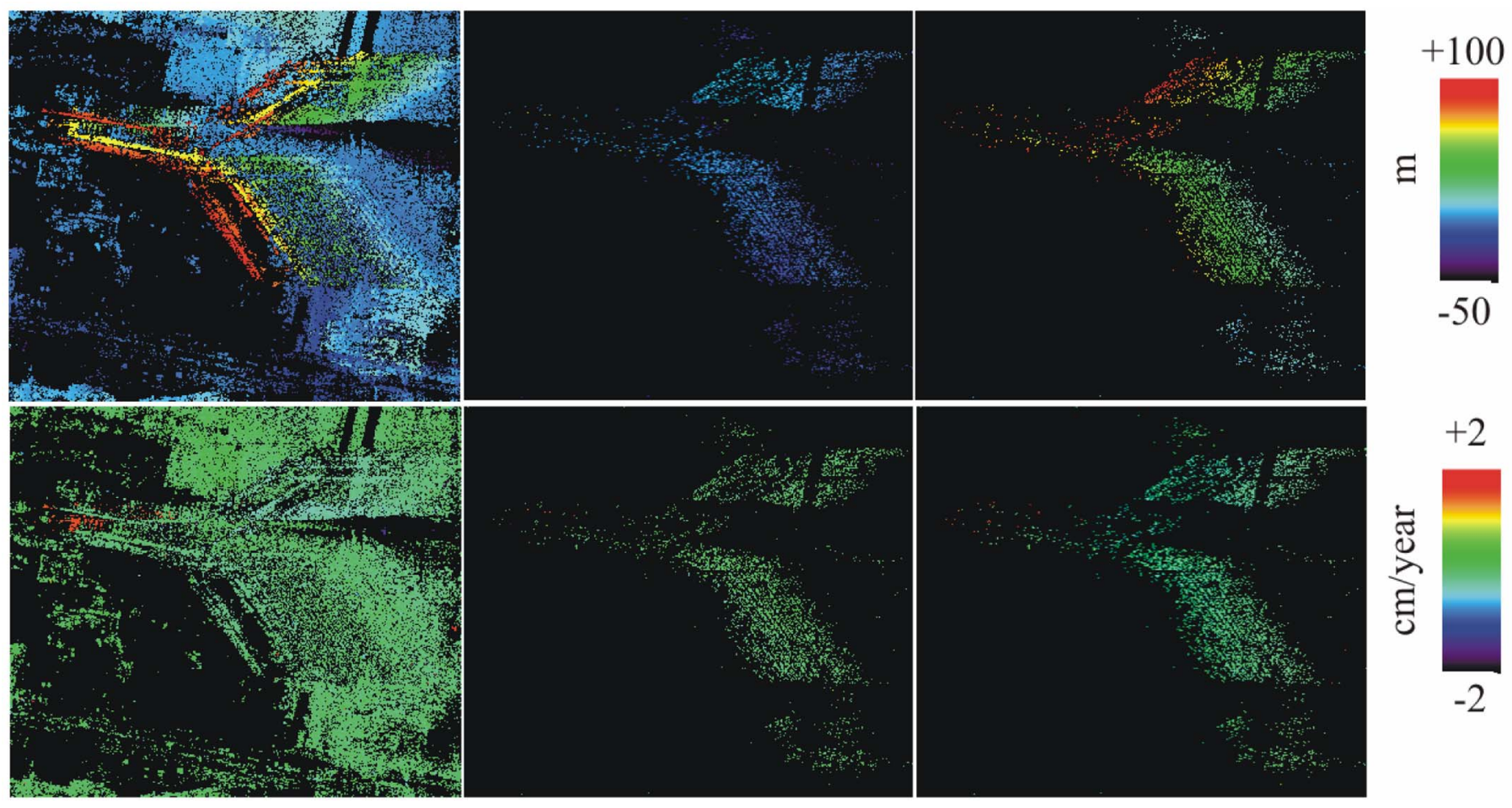

Fig. 6. (Upper row) Residual topography and (bottom row) mean deformation velocity estimated by means of the SAR tomography for (left column) single scatterers, (middle column) lower layer of double scatterers, and (right column) the upper layer of double scatterers.

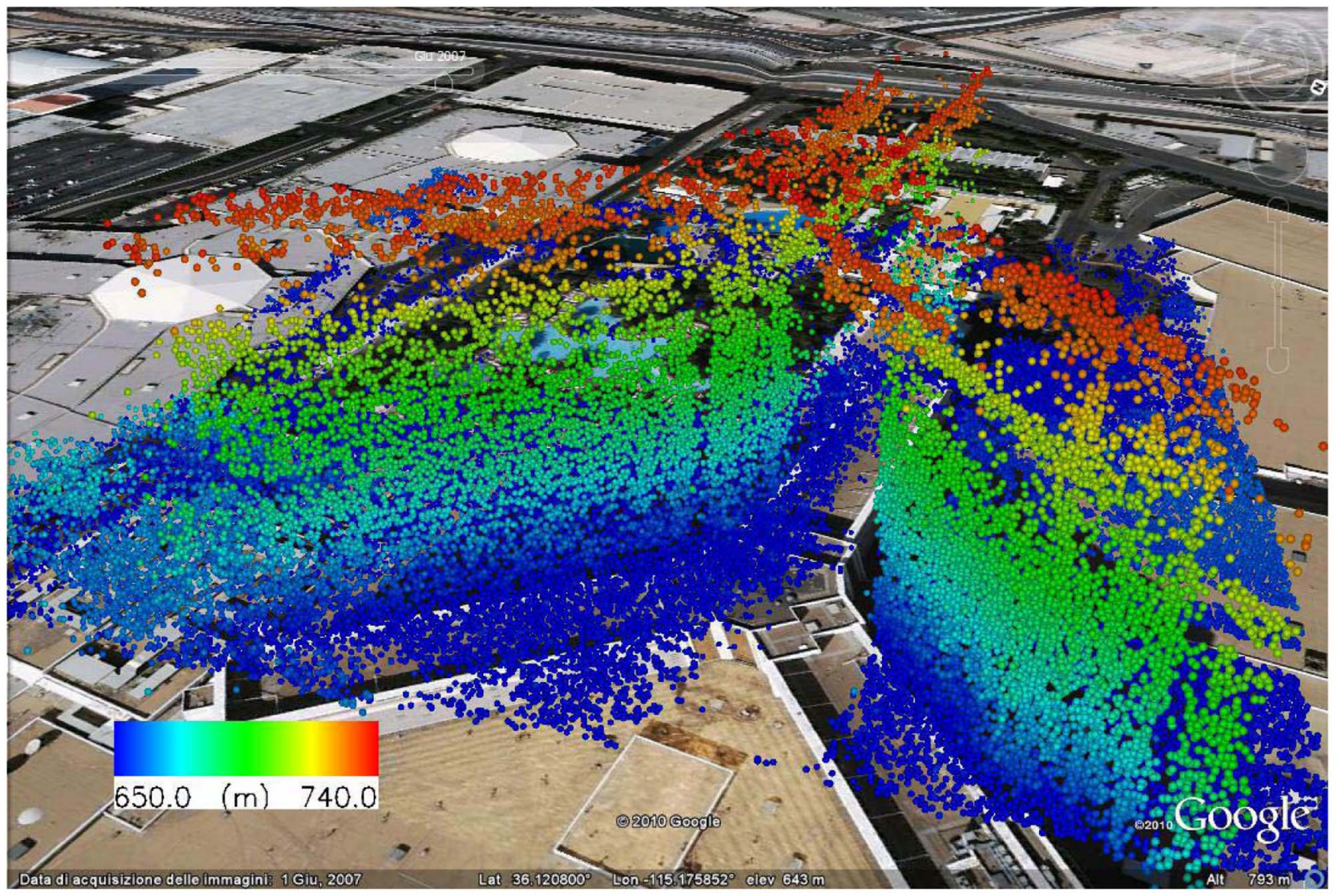

Fig. 7. Three-dimensional visualization of the single and double scatterers reconstructed with SAR tomography on Google Earth. The color is associated to the estimated height. 
can be seen, thermal dilation provides contributions leading to a mismatch with the linear displacement model that is commonly adopted in the detection of scatterers [15]. This aspect is the subject of future investigations.

\section{B. Double-Scatterer Analysis}

The assumption, made by the classical interferometric techniques, of a single scatterer per pixel neglects the interference of scatterers. We therefore applied the detection scheme described in [10] which is able to test the presence of single and double scatterers. In Fig. 6, the results of this detection algorithm are presented with the colors coded accordingly to (upper row) the estimated topography and (bottom row) mean deformation velocity. These figures show the capability of the tomographic approach to "separate" the interfering layers associated with the ground and the façade of the building. The images on the left column show the detected single scatterers, whereas in the middle and right columns, the images are associated with the ground and top layer extracted from double-scatter results, respectively. The effectiveness of tomography in scatterer pair separation on this layover (distributed over several range pixels) is particularly evident in the topography reconstruction; see the homogeneity of blue color of the ground scatterer layer and the gradation of colors on the layer corresponding to the façade. The high density of detected double scatterers that fills the lack of the single scatterers analysis should be noticed.

A further confirmation of the results is provided by the shadow stripe highlighted by the white box in Fig. 4: As expected, no double scatterers were detected in this area.

For what concerns the deformation maps shown in the right column in Fig. 6, by analyzing both the estimated mean deformation velocity and the previously estimated topography, it is interesting to note the presence of few pixels showing an estimated velocity that is fully congruent with that of the single scatterers affected by strong thermal dilation.

Finally, the 3-D view of the building is shown in Fig. 7 to demonstrate the impressive potential of the new (highresolution) sensor generation and the potential of SAR tomography for urban area analysis. It shows a 3-D view of the Mirage Hotel in Google Earth obtained with the identified single and double scatterers and without the use of the optical Google 3-D model of the building as background: The different floors are well visible in the left façade. The results show that these SAR sensors orbiting hundreds of kilometers from the Earth can provide accurate $3-\mathrm{D}$ reconstruction and monitoring of single buildings.

\section{CONCLUSION}

High-resolution SAR systems, such as TSX and CosmoSkyMed, provide an obvious improvement in the imaging capabilities. However, specific problems associated with the geometry of SAR become more evident: Layover is among them, and it affects particularly the images of urban areas. By processing spotlight TSX data, in this letter, we have shown that SAR tomography can solve this problem and allow accurate 3 -D reconstruction and monitoring. Layover associated to tall buildings and distributed over several pixels was successfully resolved.

Whereas layover is solvable by using, as shown, SAR tomography, no solutions are available for shadowing. Hence, small incidence angles are preferred for imaging urban areas to "pierce" areas with high density of buildings and reduce shadowing.

\section{REFERENCES}

[1] P. Berardino, G. Fornaro, R. Lanari, and E. Sansosti, "A new algorithm for surface deformation monitoring based on small baseline differential SAR interferograms," IEEE Trans. Geosci. Remote Sens., vol. 40, no. 11, pp. 2375-2383, Nov. 2002.

[2] A. Ferretti, C. Prati, and F. Rocca, "Nonlinear subsidence rate estimation using permanent scatterers in differential SAR interferometry," IEEE Trans. Geosci. Remote Sens., vol. 38, no. 5, pp. 2202-2212, Sep. 2000.

[3] F. Lombardini, "Differential tomography: A new framework for SAR interferometry," IEEE Trans. Geosci. Remote Sens., vol. 43, no. 1, pp. 3744, Jan. 2005.

[4] G. Fornaro, D. Reale, and F. Serafino, "Four-dimensional SAR imaging for height estimation and monitoring of single and double scatterers," IEEE Trans. Geosci. Remote Sens., vol. 47, no. 1, pp. 224-237, Jan. 2009.

[5] G. Fornaro, F. Serafino, and F. Lombardini, "3D multipass SAR focusing: Experiments with long-term spaceborne data," IEEE Trans. Geosci. Remote Sens., vol. 43, no. 4, pp. 702-712, Apr. 2005.

[6] G. Fornaro, F. Serafino, and D. Reale, "4D SAR imaging: The case study of Rome," IEEE Geosci. Remote Sens. Lett., vol. 7, no. 2, pp. 236-240, Apr. 2010.

[7] X. Zhu and R. Bamler, "Very high resolution spaceborne SAR tomography in urban environment," IEEE Trans. Geosci. Remote Sens., vol. 48, no. 12, pp. 4296-4308, Dec. 2010.

[8] S. Gernhardt S, N. Adam, M. Eineder, and R. Bamler, "Potential of very high resolution SAR for persistent scatterer interferometry in urban areas," Ann. GIS, vol. 16, no. 2, pp. 103-111, Jun. 2010.

[9] A. De Maio, G. Fornaro, and A. Pauciullo, "Detection of single scatterers in multi-dimensional SAR imaging," IEEE Trans. Geosci. Remote Sens., vol. 47, no. 7, pp. 2284-2297, Jul. 2009.

[10] A. De Maio, G. Fornaro, A. Pauciullo, and D. Reale, "Detection of double scatterers in SAR tomography," in Proc. IEEE IGARSS Conf., Cape Town, South Africa, Jul. 13-17, 2009.

[11] X. Zhu and R. Bamler, "Tomographic SAR inversion by L1-norm regularization-The compressive sensing approach," IEEE Trans. Geosci. Remote Sens., vol. 48, no. 10, pp. 3839-3846, Oct. 2010.

[12] A. Budillon, A. Evangelista, and G. Schirinzi, "Three-dimensional SAR focusing from multipass signals using compressive sampling," IEEE Trans. Geosci. Remote Sens., pp. 1-12, to be published.

[13] G. Fornaro, A. Pauciullo, and F. Serafino, "Deformation monitoring over large areas with multipass differential SAR interferometry: A new approach based on the use of spatial differences," Int. J. Remote Sens., vol. 30, no. 6, pp. 1455-1478, Apr. 2009.

[14] Average Daily Temperature Archive. [Online]. Available: http://www. engr.udayton.edu/weather/

[15] X. Zhu and R. Bamler, "Very high resolution SAR tomography via compressive sensing," in Proc. ESA FRINGE Workshop Adv. Sci. Appl. SAR Interferometry, Frascati, Italy, 2009. 